\title{
Quantitative Analysis Method of Ophthalmic Microbial Membrane Function Based on Microbiological Analysis
}

\author{
Xuanrong $\mathrm{XU}^{1 *}$, Yutong LIU² \\ ${ }^{1}$ Tianjin Medical University \\ ${ }^{2}$ Tianjin Medical University
}

\begin{abstract}
Ophthalmic microbial eye membrane is a kind of membrane complex with highly complex structure, but it also has the therapeutic effect of bacteria that can produce microbial eye membrane. Nowadays, there is no effective method to analyze the microbial membrane. Therefore, a quantitative analysis method of ophthalmic microbial membrane function based on microbiological analysis is proposed. The biomass per unit area, substrate coverage and average thickness of the biofilm were quantitatively analyzed with Staphylococcus as material and microbiological analysis method. The structure indexes such as biomass, average thickness and average diffusion distance increased significantly, indicating the transformation process of microbial membrane from occurrence to maturity. Microbiological analysis method can effectively evaluate the occurrence, development and maturation of microbial membrane, and has potential value in studying the theoretical mechanism of microbial membrane formation.
\end{abstract}

\section{Introduction}

In the natural and artificial environment, microorganisms are easy to adsorb on the surface of the eyes, and form a microbial membrane by secreting extracellular polysaccharides, proteins and nucleic acids. Microbial membrane is a kind of membrane complex with coordination, functionality and high structure, which can protect microorganisms and provide a good living environment for microorganisms ${ }^{[1]}$. Studies have shown that more than $90 \%$ of the environmental microorganisms live in the biofilm, and $80 \%$ of the bacterial diseases are related to the biofilm. It is precisely because of the protection of microbial membrane that the existing antibiotic drugs are difficult to enter the depth of microbial membrane and form effective inhibition and killing of pathogens ${ }^{[2]}$. Under the dual effects of the protection of microbial membrane and the inhibition of antibiotics, microorganisms are easy to produce specific resistance to antibiotics to resist the effect of antibiotics. Therefore, it is of great theoretical value to study the formation process of microbial membrane for the prevention and treatment of clinical pathogenic bacteria ${ }^{[3]}$. However, there are many factors affecting the formation of microbial membrane, including the physical and chemical properties of adhesion medium, the type and concentration of carbon source, osmotic pressure, water flow stress and the community composition of microbial membrane. At the same time, due to the lack of molecular microbial technology, it is difficult to study the formation mechanism of microbial membrane. The biomass per unit area, substrate coverage, average thickness and roughness coefficient of the biofilm were quantitatively analyzed by using laser scanning confocal microscopy (LSCM) imaging technology combined with microbial film analysis software. The growth process of the biofilm was studied, and the inhibitory effect of antibiotics on the biofilm was investigated. Although this analysis method can effectively evaluate the development of microbial membrane, it is not sensitive to host immune response and easy to cause infectious diseases. Therefore, a quantitative analysis method of ophthalmic microbial membrane function based on microbiological analysis was proposed.

\section{Materials, reagents, equipment and microbial culture}

\subsection{Materials and reagents}

In this experiment, $50 \mu \mathrm{L}$ of activated coagulase negative staphylococcus $(\mathrm{OD} 600=0.5)$ was added into $10 \mathrm{~mL}$ sterilized M63 medium (ammonium sulfate $0.2 \mathrm{~g}$, potassium dihydrogen phosphate $1.36 \mathrm{~g}$, ferrous sulfate heptahydrate $0.5 \mathrm{mg}$, glycerin $0.2 \mathrm{~g}$, casein hydrolysate $0.5 \mathrm{~g}$, magnesium sulfate heptahydrate $0.2 \mathrm{~g}$, glucose 0.2 $\mathrm{g}$, deionized water $100 \mathrm{~mL}$ ), and the mixture was fully shaken. In this experiment, a disposable polyethylene aseptic plate (diameter: $90 \mathrm{~m}$ ) was taken, and a sterilized cover glass was placed in the middle. The diluted bacterial liquid was added to the aseptic plate and cultured at $300 \mathrm{C}$, with attention to maintaining air humidity.After the completion of the culture time, carefully remove the cover glass (observation sample)

\footnotetext{
* Corresponding author: xuxuanrong19990828@126.com
} 
with microbial membrane with tweezers, place it in a clean culture dish, and gently wash the residual culture medium on the surface with normal saline ${ }^{[4,5]}$.

Kanamycin and Apramycin were purchased from Shanghai Jierui biological Co., Ltd. T4 DNA ligase was purchased from Thereto company. Plasmid Extraction Kit and agarose gel Recovery Kit were purchased from TIANGEN Co., Ltd. PWEB TNCTMCosmid Cloning Kit is the product of EPICENTRE company. Trypsin, yeast extract, sodium chloride, agar powder and other reagents were purchased from Shanghai Shenggong biology Co., Ltd.

\subsection{Equipment}

The test equipment is shown in Table 1.

Table 1. Test equipment.

\begin{tabular}{cc}
\hline Equipment & Manufacturer \\
\hline Gel X1520 gel imager & $\begin{array}{c}\text { Shanghai Ouxiang Scientific } \\
\text { Instrument Co., Ltd }\end{array}$ \\
$\begin{array}{c}\text { CT15RT high speed } \\
\text { freezing centrifuge }\end{array}$ & Shanghai Tianmei \\
UV-2600 ultraviolet & \\
visible & Shimadzu Corporation of Japan \\
spectrophotometer & \\
GYY-6C constant & \\
voltage and current & Beijing 61 Biotechnology Co., \\
electrophoresis & Ltd \\
instrument & \\
LDZM-40KOS vertical \\
pressure steam sterilizer \\
SW-CJ-IFD ultra clean \\
worktable & Shanghai Shenan Medical \\
HYG-C shaker & Equipment Co., Ltd \\
SL2001N electronic & Taicang experiment purification \\
balance & factory \\
LC-20A high & Scientific Instrument \\
performance liquid & Shimadzu Corporation of Japan \\
chromatography &
\end{tabular}

In order to facilitate the experiment, an ophthalmic bacteria specimen preparation table is provided, which includes a working table, a placing box, a preparation table and a drawer. The two sides of the bottom end of the preparation table are threaded with table legs, the bottom end of the table legs is embedded with anti slide, and the middle of the table legs is movably provided with a drawer. A glass is fixed on both sides of the internal thread of the drawer, an ultraviolet lamp is installed on the internal measurement of the glass, a placement box is fixed on one side of the surface thread of the preparation table, and a placement bucket is set inside the placement box ${ }^{[6-9]}$. The other side of the preparation table is provided with a roller groove, the bottom end of the inner side of the roller groove is provided with a limiting groove, the inner part of the roller groove is provided with a sliding roller, and the middle top end of the roller is provided with a first placing groove ${ }^{[10]}$. A worktable is welded in the middle of the surface of the preparation table, a sliding plate is arranged inside the sliding plate, a second placing groove is arranged on both sides of the surface of the sliding plate, and a push plate is fixed on one side of the sliding plate ${ }^{[11]}$.

The ophthalmic bacteria and microorganism specimen preparation table is shown in Figure 1.

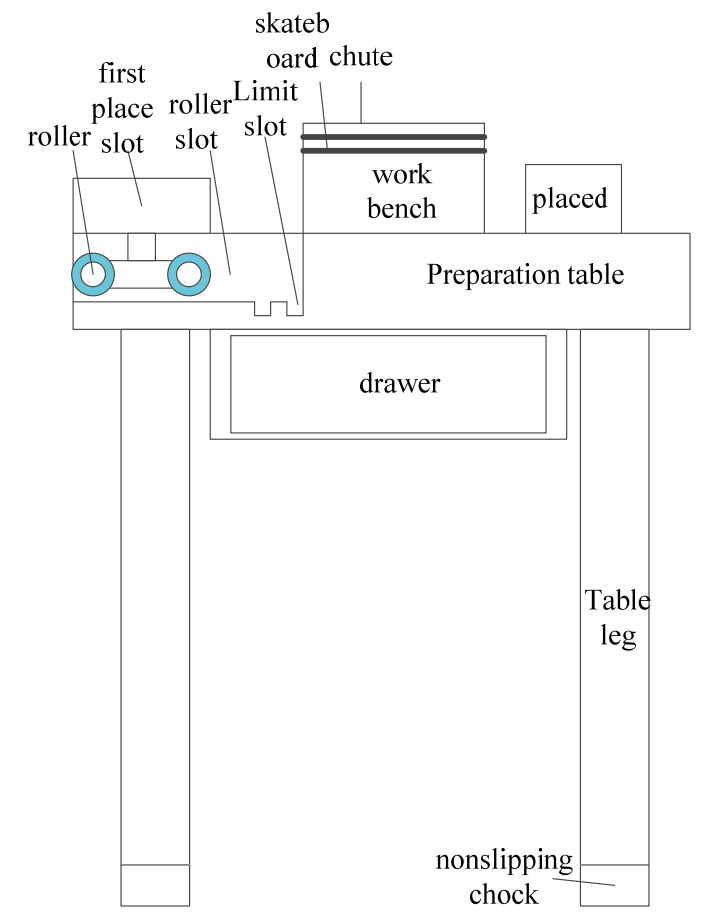

Fig. 1. Ophthalmic bacteria and microorganism specimen preparation table.

It can be seen from Figure 1 that the first placing groove can be moved by the coordination of the roller and the roller groove, so as to achieve the effect of removing the first placing groove when the alcohol lamp is not used, which is convenient for the user to operate. By setting the first placing groove, the alcohol lamp can be placed inside, so as to achieve the effect of providing the placement position.

By setting the limit slot, the alcohol lamp in the first place slot is fixed close to the workbench, and the effect of limit fixing is achieved to prevent sliding away. By the cooperation of slide groove and slide plate, the slide slide placed in the second placement groove can move horizontally, and the effect of drying can be achieved by moving to alcohol lamp.

By setting a second placing groove, the slide can be placed inside, so as to provide a placement position and embed and fix the slide. Through the cooperation of the placing box and the placing bucket, the work needed by the staff can be inserted and placed, so as to provide the placement position and facilitate the use.

By setting drawers, the clothes and gloves that the staff need to wear can be placed inside, so as to achieve the effect of providing the placement position. Through the ultraviolet lamp, the clothes and gloves placed in the drawer can be sterilized, so as to achieve the effect of sterilization at the same time. 


\subsection{Preparation of ophthalmic microbial film samples}

Preparation of dye: take clean EP tube, wrap it tightly with tin foil, add $1 \mathrm{~mL}$ DMSO to it, melt the fluorescent dye LIVE / DEAD BaclightTM Bacterial Viability Kit at room temperature, add $1.5 \mu \mathrm{L}$ SAT09 dye into DMSO, shake and mix well.

Add $200 \mu \mathrm{L}$ dye to the cover glass to ensure that the whole sheet is covered with dye solution. Pack the Petri dish with cover glass with tin foil, dye for 20-30 min, take out the cover glass and suck up the liquid at the edge. Add $10 \mu \mathrm{L}$ of sealing agent to the clean slide, place the stained surface of the cover glass plate downward, press it down, and remove the bubbles to ensure that the vision is clear and does not flow. The prepared films were wrapped with tin foil and observed by laser scanning confocal microscope.

\subsection{Sample observation}

For the first time, open the laser scanning confocal microscope according to the corresponding sequence, enter the operation software FV10, select the corresponding dye options, and adjust the appropriate excitation range. Secondly, select a 100 times oil mirror, drop oil, place the dyed film upside down, select the light source, adjust the thickness of the quasi focus screw to obtain a clear field of vision. Thirdly, switch the field of view to the scanning interface, adjust the scanning speed to the maximum, adjust the brightness and other parameters to obtain a clear scanning interface, and rotate the fine quasi focus screw to determine the upper and lower bottom surface parameters of the sample. After that, the scanning speed is adjusted to 20 times, and Z-axis scanning is performed to obtain a group of sample images. Finally, the image is saved and converted to a 3D image accordingly.

The formation process of coagulase negative Staphylococcus biofilm is shown in Figure 2.

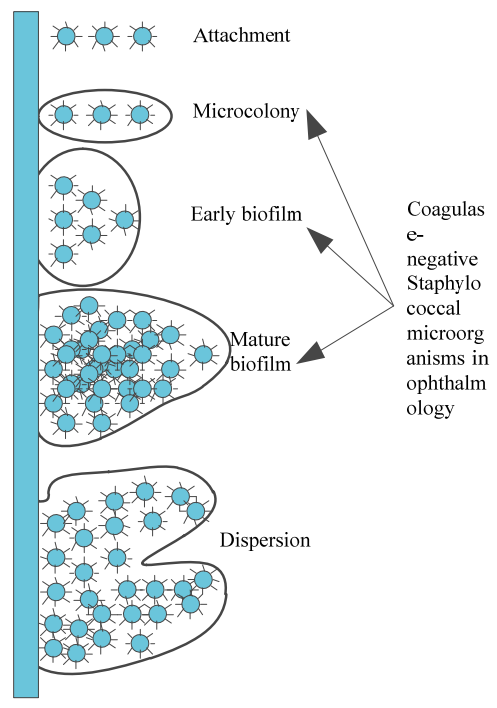

Fig. 2. Biofilm formation of coagulase negative Staphylococcus in ophthalmology

\section{Quantitative analysis of microbial membrane function based on microbiological analysis}

\subsection{Construction of metagenomic library}

The cultured samples were sieved through different pore size sieves, and the total DNA of microorganisms was directly extracted by lysate and SDS buffer. After the concentration and purity were determined by electrophoresis, the DNA terminal was modified and leveled by the terminal repair enzyme of pWEB TNCTM Cosmic Cloning Kit, and then connected with the prepared flat terminal shuttle expression vector pJTU2554. The ligation products were packaged with phage protein and then infected with the host bacteria of the library. $10 \mu \mathrm{L}$ of the infection mixture was spread on the LB plate containing $50 \mu \mathrm{g} / \mathrm{mL}$ ampicillin, cultured overnight at $37{ }^{\circ} \mathrm{C}$, and the titer was counted and calculated. According to the calculated titer, the obtained library bacteria were sub packed into the culture tube, each tube contained about 500 clones, which were cultured overnight at $37^{\circ} \mathrm{C}$ for standby.

\subsection{Screening of positive clones}

The library bacteria cultured overnight were inoculated into the new LB medium until $\mathrm{OD}_{600}$ reached 0.4-0.8 (growth state), and then mixed with Streptomyces albicans. Then, the mixed bacteria solution was evenly coated on the MS solid plate containing $10 \mathrm{mmol} / \mathrm{L}$ $\mathrm{MgCl} 2$ and incubated at $30{ }^{\circ} \mathrm{C}$ for $16-18 \mathrm{H}$. Subsequently, the aqueous solution containing nalidixic acid $(0.5 \mathrm{mg} / \mathrm{mL})$ and Apramycin $(0.4-0.5 \mathrm{mg} / \mathrm{mL})$ was coated and cultured for 3-5 days. The zygotes were photocopied on solid medium R5, MS and ISP4 with sterilized gauze. After 5-7 days of continuous culture, the upper layer was covered with soft solid medium containing $0.6 \%-0.7 \%$ agar containing Staphylococcus aureus. After the upper agar was coagulated and cultured at $30{ }^{\circ} \mathrm{C}$ for $1-2$ days, observe whether there is a transparent inhibition zone in the upper agar. If there is a transparent inhibition zone, select the clone and scribe again to verify its antibacterial activity.

\subsection{Positive clone fermentation and product extraction}

Different media were used to ferment the positive clones. By optimizing the types of media and fermentation time, MS and ISP4 (containing resin) were selected as the fermentation media to achieve the highest activity. Take a certain amount of dry sample, add purified water, boil for 2 hours, filter with gauze, collect the filtrate, add DMannitol to dissolve, and prepare MS liquid medium. After the volume is fixed, it will be packed separately. Each $250 \mathrm{~mL}$ conical flask was sub packed with $50 \mathrm{~mL}$ medium, sealed with 8 layers of gauze, and then sterilized at high temperature. Ampicillin was added according to the final concentration of $50 \mu \mathrm{g} / \mathrm{mL}$ and 
inoculated with baozi. The cells were cultured at 225 $\mathrm{r} / \mathrm{min}$ and $28^{\circ} \mathrm{C}$ for 3 days. The medium turned brown, and was transferred to isp4 (resin content $3 \%-5 \%$ ) medium at $1 \%$ ratio, $225 \mathrm{r} / \mathrm{min}$, and cultured at $28{ }^{\circ} \mathrm{C}$ for 12 days. The resin adsorbs the fermentation product, washes the culture medium, recovers the resin, and after drying, dissolves the product on the adsorption resin with methanol, and collects the product after rotary steaming for standby. The fermentation products of Streptomyces albus were used as the control group.

\subsection{Liquid chromatography tandem mass spectrometry for rapid detection}

Methanol was selected as the extraction solvent, and HLB column solid phase extraction method was used to extract the sample solution and eluent of microbial membrane to eliminate the interference of matrix. According to the set screening conditions, the chromatogram of microbial membrane was analyzed to complete the rapid detection of microbial membrane.

\subsubsection{Extraction solvent selection}

For some samples with high degree of reaction, ethyl acetate was directly used for extraction. The dispersity of the sample is very poor and the extraction effect is not ideal. To do this, add $5 \mathrm{~mL}$ of water, $5 \%$ ammonia and $1 \%$ acetic acid to the sample and mix well. Although using methanol, acetonitrile, acetone and ethyl acetate as extractants has good recovery effect, due to different chemical properties, the solubility of impurities in the solution also has great differences, especially in the complex environment, the matrix difference is great. Therefore, methanol was selected as the extraction solvent.

\subsubsection{Solid phase extraction}

The matrix can not be eliminated completely by using methanol as the extractant. Therefore, the solid phase extraction method is used to further purify. Because of the different internal structure of some microorganisms, the HLB column and CIA column can obtain the ideal recovery rate. However, the impurity removal effect of CIA column is not as good as that of HLB column. Using silica gel column purification, polar impurities can not be well removed, which greatly interferes with the detection of polar substances and can not get the ideal recovery rate. Therefore, HLB column purification is selected. HLB column solid phase extraction method was used to extract the sample solution and eluent of microbial membrane. Methanol water was mixed in the ratio of $1: 1 / 2: 3 / 3: 7 / 1: 4$, and determined in the ratio of $3: 2 / 3: 8 / 2: 9$ and $1: 10$. The recovery of microbial membrane was obtained.

\subsubsection{Microbial membrane chromatography}

Methanol solution, methanol and $0.1 \%$ formic acid mixed solution, acetonitrile solution, acetonitrile and
$0.1 \%$ formic acid mixed solution were used as the detection conditions of mobile relative microbial membrane. The chromatogram of microbial membrane is shown in Figure 3.

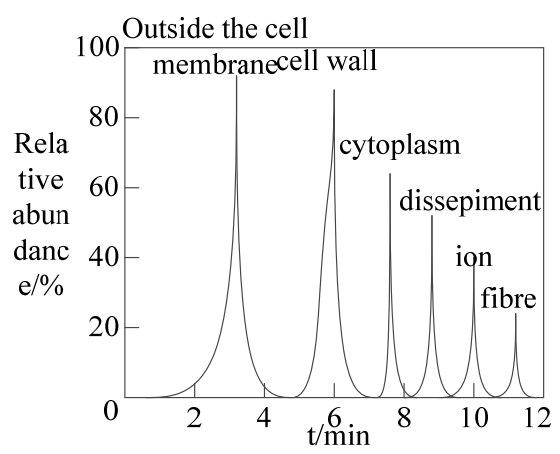

Fig. 3. Chromatogram of microbial membrane.

The results showed that the reaction of each analyte was stable with methanol as the organic phase, and the peak shape was good, while the reaction volume was significantly reduced with acetonitrile as the organic phase. When formic acid was added to the mobile phase, the ionization efficiency and sensitivity of the target compound could be improved. Methanol- $0.1 \%$ formic acid aqueous solution was used as the mobile phase. According to the chromatogram shown in Figure 3, the biofilm can be quickly detected.

\section{Result}

\subsection{The growth of biofilm was observed}

After 4 days of cultivation, the growth of microbial membrane is shown in Figure 4.

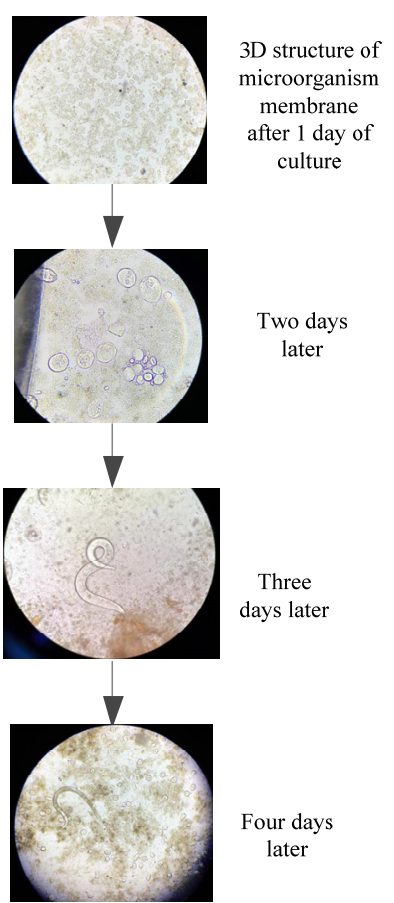

Fig. 4. Growth of microbial membrane. 
It can be seen from Figure 4 that the biomass of microbial membrane presents an obvious growth trend, and shows a structured microbial membrane morphology.

The microbiological analysis software can be used to analyze a number of microbial membrane structure indexes. The specific contents and meanings are summarized in Table 2.

Table 2. Structure index of microbial coating.

\begin{tabular}{cc}
\hline Data indicators & Meaning \\
\hline Biomass & $\begin{array}{c}\text { It represents the overall volume of the } \\
\text { biofilm. It can also be used as an indirect } \\
\text { measure of the number of } \\
\text { microorganisms per unit area. }\end{array}$ \\
$\begin{array}{c}\text { It reflects the efficiency of microbial } \\
\text { colonization on the substrate surface. }\end{array}$ \\
$\begin{array}{c}\text { Average } \\
\text { thickness }\end{array}$ & $\begin{array}{c}\text { It provides a method for measuring the } \\
\text { size of microbial membrane space. }\end{array}$ \\
$\begin{array}{c}\text { Fractional } \\
\text { dimension }\end{array}$ & $\begin{array}{c}\text { It is used to measure the complexity of } \\
\text { microbial membrane structure. }\end{array}$ \\
$\begin{array}{c}\text { It can be used to measure the degree of } \\
\text { coefficient } \\
\text { change in the thickness of biofilm, and } \\
\text { also to indicate the degree of } \\
\text { heterogeneity of biofilm. }\end{array}$ \\
$\begin{array}{c}\text { Average } \\
\text { diffusion } \\
\text { distance }\end{array}$ & $\begin{array}{c}\text { It represents the diffusion distance } \\
\text { between the nutrient source and the } \\
\text { microorganism in the biofilm. }\end{array}$ \\
$\begin{array}{c}\text { It reflects the proportion of microbial } \\
\text { Ratio of surface } \\
\text { area to biomass }\end{array}$ & $\begin{array}{c}\text { membrane contacting nutrient sources and } \\
\text { the ability of microbial membrane to } \\
\text { adapt to the environment. }\end{array}$ \\
\hline
\end{tabular}

The biofilm growth of Staphylococcus was investigated in terms of biomass, average thickness, roughness coefficient, average diffusion distance, surface area to biomass ratio, as shown in Table 3 .

Table 3. Statistics of growth characteristics of microbial film.

\begin{tabular}{ccccc}
\hline $\begin{array}{c}\text { Culture } \\
\text { days / day }\end{array}$ & $\mathbf{1}$ & $\mathbf{2}$ & $\mathbf{3}$ & $\mathbf{4}$ \\
\hline Biomass & $1.45 \pm 0.005$ & $2.65 \pm 0.20$ & $6.75 \pm 3.50$ & $10.25 \pm 1.65$ \\
$\begin{array}{c}\text { Average } \\
\text { thickness }\end{array}$ & $1.65 \pm 0.35$ & $3.25 \pm 0.75$ & $8.05 \pm 0.45$ & $9.25 \pm 1.66$ \\
$\begin{array}{c}\text { Roughness } \\
\text { coefficient }\end{array}$ & $0.75 \pm 0.15$ & $0.50 \pm 0.05$ & $0.10 \pm 0.05$ & $0.11 \pm 0.05$ \\
$\begin{array}{c}\text { Average } \\
\text { diffusion } \\
\text { distance } \\
\begin{array}{c}\text { Ratio of } \\
\text { surface } \\
\text { area to } \\
\text { biomass }\end{array}\end{array}$ & $0.15 \pm 0.02$ & $0.22 \pm 0.01$ & $0.85 \pm 0.25$ & $3.45 \pm 0.76$ \\
\hline
\end{tabular}

According to Table 3, the biomass of Staphylococcus increased from 1.47 (the first day) to $10.33 \mu \mathrm{m} 3 / \mu \mathrm{m} 2$ (the fourth day) with the growth of culture time. At the same time, the average thickness of microbial membrane increased gradually with the increase of culture days, reaching the maximum value of $9.26 \mu \mathrm{m}$ on the fourth day. In terms of the average diffusion distance, it showed a slow growth trend in the first three days, which were $0.14 \mu \mathrm{m}, 0.21 \mu \mathrm{m}$ and $0.83 \mu \mathrm{m}$ respectively, and increased rapidly in the fourth day, reaching $3.41 \mu \mathrm{m}$. However, the roughness coefficient, surface area and biomass ratio of staphylococcal biofilm were negatively correlated with the culture days.

\subsection{Inhibitory effect of antibiotics on the growth of biofilm}

The results of 3D imaging and plane map showed that compared with the negative control, the growth of staphylococcal biofilm treated with $10 \mu \mathrm{g} / \mathrm{mL}$ and 100 $\mu \mathrm{g} / \mathrm{mL}$ kanamycin was significantly inhibited, and the inhibition effect increased with the increase of kanamycin concentration.

The formula of inhibition rate is as follows:

$$
(P \%)=\left(A_{2}-A_{1}\right) /\left(A_{2}-A_{0}\right) \times 100
$$

In formula (1), $A_{0}$ is the blank control group; $A_{1}$ is the sample action group; $A_{2}$ was negative control group.

In order to further investigate the inhibitory effect of Kanamycin on Staphylococcus biofilm, COMSTAT software was used to analyze the biomass, average thickness, average diffusion distance and the ratio of surface area to biomass of the biofilm. The results are shown in Figure 5.
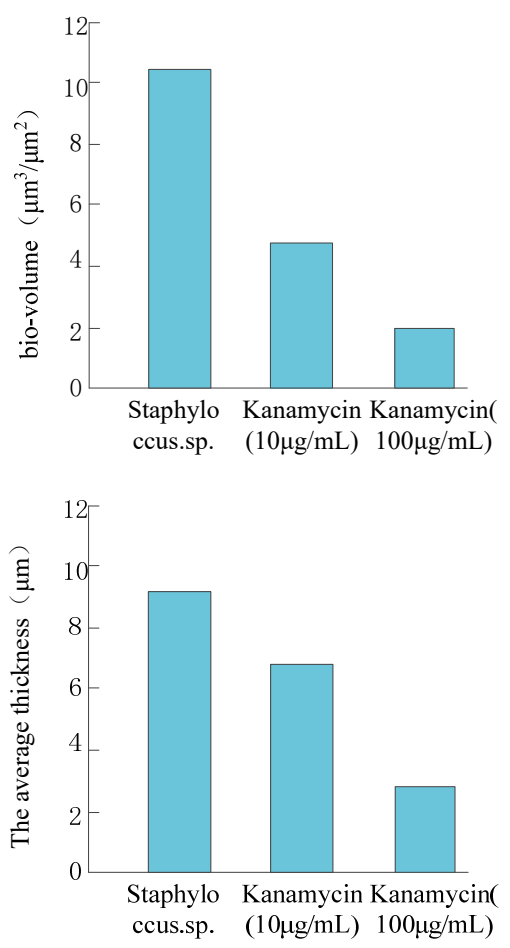


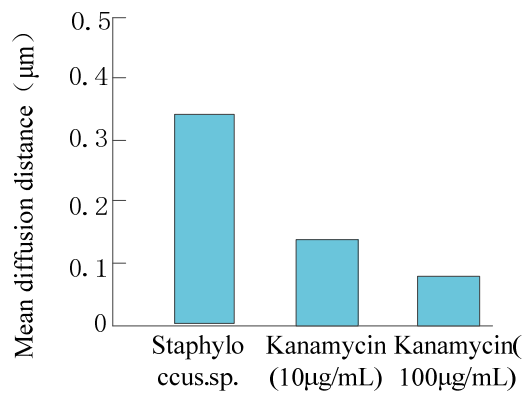

Fig. 5. Spatial structure data of kanamycin inhibiting microbial membrane.

With the addition of kanamycin, the biomass, average thickness and average diffusion distance of the biofilm decreased, and there was a negative correlation with the increase of antibiotic concentration, which indicated that antibiotics reduced the number of microorganisms in the biofilm to a certain extent and damaged the structure of the biofilm. However, the ratio of surface area to biomass increased with the increase of kanamycin concentration, which also reflected the obvious changes in the morphology of staphylococcal biofilm under the interference of antibiotics, showing a certain ability to adapt to the environment.

\section{Conclusion}

In conclusion, the indicators of microbiological analysis can effectively reflect the growth and change process of the whole microbial membrane, which fully shows that the quantitative analysis of microbial membrane based on microbiological analysis has potential value in the study of the theoretical mechanism of microbial membrane formation, and can provide a theoretical basis for the study of the pathogenicity of microbial membrane. One day in the future, people can use microorganisms to treat eye diseases through research.

\section{References}

1. Y . Sato., Y. J. Zhao, T. Hori, et al. Transition of microbial community structures after development of membrane fouling in membrane bioreactors (MBRs)[J]. AMB Express, 10(18):1-10(2020).

2. N. J. Kumar, B. George, M. Sivaprakasam. A Membrane-Potentiometer-Based Palpation Position Sensor Suitable for Ophthalmic Anesthesia Training[J]. IEEE Sensors Journal, 20(6):33243332(2020).

3. J. Teng, M. Zhang, K. T. Leung, et al. A unified thermodynamic mechanism underlying fouling behaviors of soluble microbial products (SMPs) in a membrane bioreactor[J]. Water Research, 149(2):477-487(2019).

4. E . Grau, F. Horn, U. Nixdorff, et al. OCT and IOP findings in a healthy worker cohort: results from a teleophthalmic study in occupational medicine[J]. Graefe's Archive for Clinical and Experimental Ophthalmology, 257(11):2571-2578(2019).
5. A. Sarimsakov, A . Shukurov, K. Yunusov, et al. Drug Delivery Polymer Systems for Ophthalmic Administration of Anti-Viral Agents[J]. Current Drug Delivery, 17(5):406-413(2020).

6. S. W. Du, C. Sun, A. Q. Ding, et al. Microbial dynamics and performance in a microbial electrolysis cell-anaerobic membrane bioreactor[J]. Journal of Zhejiang University - Science A: Applied Physics \& Engineering, 20(7):533-545(2019).

7. V. Razaviarani, M. Ruiz-Uriguen, P. R. Jaffe. Denitrification of Nitric Oxide Using Hollow Fiber Membrane Bioreactor; Effect of Nitrate and Nitric Oxide Loadings on the Reactor Performance and Microbiology[J]. Waste \& Biomass Valorization, 10(7):1989-2000(2019).

8. R . Rossi, X. Wang, B. E. Logan. High performance flow through microbial fuel cells with anion exchange membrane[J]. Journal of Power Sources, 475:228633(2020).

9. K . Beblo-Vranesevic, M . Bohmeier, A. K. Perras, et al. The responses of an anaerobic microorganism, Yersinia intermedia MASE-LG-1 to individual and combined simulated Martian stresses[J]. PLoS ONE, 12(10):178-185(2017)

10. M. G. Mehta-Kolte, D. Loutey, O. Wang, et al. Mechanism of H2S Oxidation by the Dissimilatory Perchlorate-Reducing Microorganism Azospira suillum PS[J]. mBio, 8(1):16-23(2017).

11. A. Yoshihara, T. Kozakai, T. Shintani, et al. Purification and characterization of d-allulose 3epimerase derived from Arthrobacter globiformis M30, a GRAS microorganism[J]. Journal of Bioscience \& Bioengineering, 123(2):170176(2017). 